\title{
Estimación in vivo de la canal porcina por el método de ulltrasonografía: Un Enfoque de la Ecointensificación en Bioeconomía Porcina
}

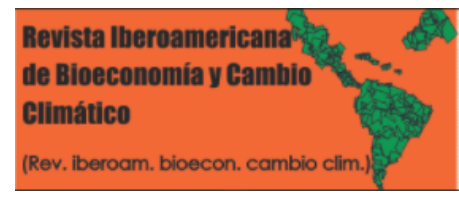

\author{
In vivo estimation of the pig carcass by the ultrasonography \\ method: An Approach to Eco-Intensification in Porcine \\ Bioeconomics
}

\author{
Hernández Zapata, Santiago; Blanco Roa, Noel; Chavarría Rivaz, \\ Elvin; Zúniga-González, Carlos; Editor Académico Prof. M.Sc Edgar \\ Marineros-Orantes
}

\author{
Santiago Hernández Zapata \\ nblanco@ev.unanleon.edu.ni \\ Empresa NICALIT, Nicaragua \\ Noel Blanco Roa \\ nblanco@ev.unanleon.edu.ni \\ Escuela de ciencias agrarias y veterinarias - UNAN - \\ León, Nicaragua \\ Elvin Chavarría Rivaz, \\ nblanco@ev.unanleon.edu.ni \\ Empresa NICALIT, Nicaragua \\ Carlos Zúniga-González \\ czuniga@ev.unanleon.edu.ni \\ Escuela de ciencias agrarias y veterinarias - UNAN - \\ Le, Nicaragua \\ Editor Académico Prof. M.Sc Edgar Marineros- \\ Orantes \\ Universidad de El Salvador, El Salvador
}

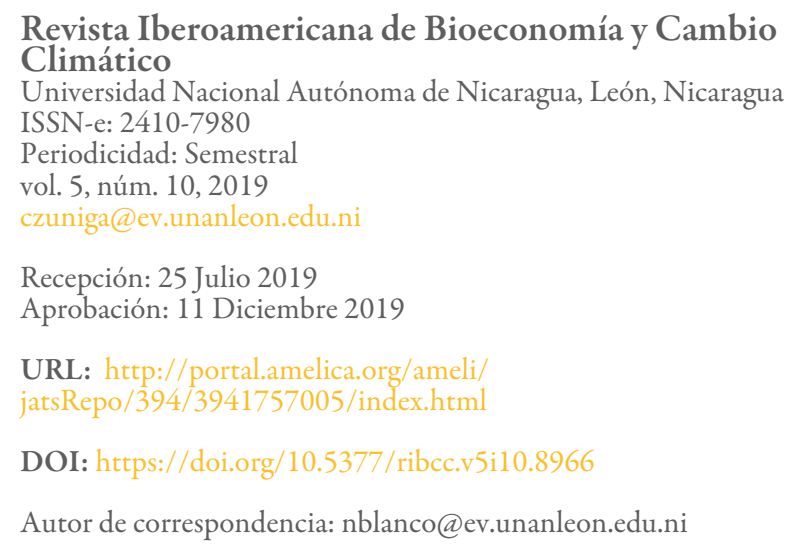

Revista Iberoamericana de Bioeconomía y Cambio

Universidad Nacional Autónoma de Nicaragua, León, Nicaragua ISSN-e: 2410-7980

Periodicidad: Semestral

vol. 5, núm. 10, 2019

Recepción: 25 Julio 2019

URL: http://portal.amelica.org/ameli

jatsRepo/394/3941757005/index.htm

Autor de correspondencia: nblanco@ev.unanleon.edu.ni
Resumen: En este estudio se analiza la relación entre el espesor de grasa subcutánea (EGS), espesor muscular (EM) y espesor de grasa subcutánea y espesor muscular juntos (EGSM), medidos con ultrasonido en un solo punto anatómico de cerdos vivos y los parámetros más importantes de la canal (Peso vivo, Peso de la canal, carne clase A, B, C que corresponden a las carnes de primera, segunda y tercera categoría, y cortes específicos cómo lomo, posta de pierna y aguja). En nuestro experimento utilizamos cerdos híbridos de las razas Pietrain, Landrace y Yorkshire en cantidad de 25 animales de ambos sexos. Los resultados del estudio sugieren que los parámetros de la composición de la canal aquí analizados (Peso vivo, peso de la canal, carne clase A, B, C y cortes específicos, lomo, posta de pierna y aguja) están altamente correlacionados con el espesor de la grasa subcutánea medida con el ultrasonido en la última costilla del musculo longuísimo dorsal en el lado izquierdo del cerdo vivo $(\mathrm{r}=0.53-0.67)$. La correlación múltiple del modelo de predicción de regresión lineal entre los parámetros de la canal y el espesor de grasa dorsal fue (0.74) y el coeficiente de determinación mostró suficiente capacidad de predicción (R2 $=0.55)$. Los modelos de predicción de regresión lineal de los componentes de la canal con el espesor muscular obtuvieron baja capacidad de predicción $(\mathrm{R} 2=0.23)$. Igualmente en el modelo de regresión del espesor de grasa subcutánea y espesor muscular juntos ( R2 = 0.25). Entre las correlaciones más importantes encontradas están: lomo derecho, carne clase A $(r=0.80)$, peso vivo y canal entera $(r=0.85)$ paleta derecha y carne clase $B(r$ $=0.88$ ) siendo este patrón repetitivo en todas las correlaciones de las mediciones ultrasonografica y los componentes de la canal. Los resultados obtenidos en el presente trabajo alientan su utilización como técnica predictoria de la composición de la canal porcina en el sendero productivo Ecointensificación de la Bioeconomia.

Palabras clave: Predicción, Canal, Porcino, Espesor muscular, Ecointensificacion.

Abstract: In this study we analyze the relationship between subcutaneous fat thickness (EGS), muscle thickness (MS) 
and subcutaneous fat thickness and muscle thickness together (EGSM), measured with ultrasound at a single anatomical point of live pigs and more parameters important of the carcass (live weight, carcass weight, meat class A, B, C corresponding to first, second and third category meats, and specific cuts such as loin, leg and needle post). In our experiment we used hybrid pigs of the Pietrain, Landrace and Yorkshire breeds in an amount of 25 animals of both sexes. The results of the study suggest that the parameters of the channel composition analyzed here (Live weight, carcass weight, meat class A, B, C and specific cuts, loin, leg post and needle) are highly correlated with the thickness of subcutaneous fat measured with ultrasound on the last rib of the very long dorsal muscle on the left side of the live pig $(\mathrm{r}=$ $0.53-0.67)$. The multiple correlation of the linear regression prediction model between the parameters of the carcass and the thickness of the dorsal fat was (0.74) and the coefficient of determination showed sufficient predictability $(\mathrm{R} 2=0.55)$. The linear regression prediction models of the carcass components with muscle thickness obtained low predictability $(\mathrm{R} 2=0.23)$. Similarly in the regression model of subcutaneous fat thickness and muscle thickness together $(\mathrm{R} 2=0.25)$. Among the most important correlations found are: right loin, class $\mathrm{A}$ meat $(\mathrm{r}=$ $0.80)$, live weight and whole carcass $(r=0.85)$ right shoulder and class $\mathrm{B}$ meat $(\mathrm{r}=0.88)$ being this repetitive pattern in all correlations of ultrasonographic measurements and channel components. The results obtained in this work encourage its use as a predictive technique of the composition of the pig carcass in the Ecointensification productive path of the Bioeconomy.

Keywords: Prediction, Canal, Pigs, Muscle thickness, Ecointesification.

\section{INTRODUCCIÓN}

La bioeconomía porcina se perfila como el gran actor de la bioeconomía global. El 75\% de la producción y el consumo global de cerdo se concentra en tres regiones: China, Unión Europea y Estados Unidos. Han crecido significativamente incrementándose en más de un $80 \%$ en los últimos 30 años. Por eso la Eco intensificación es referida a las prácticas agronómicas y pecuarias dirigidas a mejorar el rendimiento ambiental de las actividades agrícolas y pecuarias sin sacrificar los niveles de producción / productividad existente. Un indicador clave es la siembra directa, así podemos mencionar, por lo menos al 2006 a Brazil, Argentina, Paraguay, Bolivia, Venezuela, Chile y Colombia, según datos de la FAO evidencia esta afirmación (Dios, 2015). Más de 50 años de investigación y desarrollo han transcurrido en la calificación y clasificación de canales de cerdo. Iniciando con evaluaciones visuales, pasando por medidas directas de varios parámetros de grasa y magro con plantillas y reglas metálicas, la industria ha llegado al punto donde varias técnicas electrónicas altamente sofisticadas están disponibles (Ultrasonidos), Blanco Roa N., huba J., Hetenyi L., Oravcová A. (2008).

La canal representa el producto final de la producción del ganado porcino. De ahí que determinar la composición de una canal sea de gran importancia para muchos campos de la producción animal, pero

\section{NotAS DE AUTOR}


muy particularmente para el mejoramiento genético. Los sistemas de clasificación de canales actualmente utilizados califican la conformación, pero están muy pocos relacionados con la composición real de estas por lo que pierden objetividad y se tornan insuficientes. En la actualidad, el único medio eficaz con el que se cuenta para determinar con exactitud la composición de una canal es el despiece. No obstante, este requiere del sacrificio de los animales, inversiones en medios técnicos y fuerza de trabajo, también implica pérdida de tiempo. Esto significa que carecemos de un sistema óptimo capaz de establecer con exactitud la composición de la canal a un costo y tiempo admisible (López G., Rubio M. (1998), Londoño J., Velásquez C. (2013), Gérard D.(2001), Swantek P.; Crenshaw J.; Marchello M.; Lukaski H.(1992), Boland M.; Foster K.; et. al (1995), Ordenes L. (2005), Higbie A.; et,al (2002).

Este hecho estimula la búsqueda de nuevos y modernos sistema de clasificación y predicción de la composición de la canal. La posible solución a esta problemática, puede estar en las tecnologías modernas. La ultrasonografía como posible técnica de estimación de la canal ha sido objeto de estudio durante décadas por muchos autores tales como; C. A. Mejía, M. M. Bermúdez, P. A. Velázquez, M. Izquierdo, J. A. Cuaron y G. Daumas.(1999)

Todos correlacionaron ya sea espesor muscular, espesor de la grasa dorsal, área del musculo longuísimo dorsi medidos con ultrasonidos, como indicadores de la composición de la canal. Obteniendo resultados alentadores sobre todo en los últimos trabajos de investigación, debido principalmente a la depuración de la técnica y a la precisión de la tecnología moderna.

El objetivo principal de este estudio fue analizar el espesor muscular y grasa dorsal, como posibles predictores de la composición de la canal porcina. Establecer con bastante precisión el peso de la canal, así como el peso de la carne de primera, segunda y tercera clase, para hacer más claras las transacciones de oferta y demanda con miras a crear esquemas de pago por calidad de canal y no por el cerdo vivo solamente. Por primera vez en Nicaragua se realiza un esfuerzo de esta naturaleza, orientado a optimizar las relaciones comerciales entre productores y comerciantes (ESCI (2015), Central American Data (2015), UCO (2015), tratando de establecer un precio justo del cerdo en pie acorde a la composición de su canal. Además la estimación in vivo de la canal es un elemento de vital importancia en la mejora genética porcina para potenciar y acelerar el progreso genético futuramente (Universo porcino (2015).

\section{MATERiAl y MÉtodo}

Este estudio inició en el mes de octubre del año 2014 y finalizó en julio del año 2015. En el experimento fueron utilizados cerdos híbridos de las razas: Pietrain, Landrace y Yorkshire en cantidad de 25 animales (Hembras y Machos castrados). Los animales procedían de una sola granja porcina de la Finca NICALIT ubicada en el Km 69 en el Municipio de León en el Departamento de León-Nicaragua.

A la edad aproximada de 5 meses fueron seleccionados los 25 cerdos donde hasta la hora de realizar el ultrasonido se mantuvieron alojados en corrales de cemento, los cuales contaron con comederos tipo tolva y bebederos de chupón. Los animales fueron alimentados durante todo el experimento dos veces al día, con una dieta a base de concentrado de engorde a ración de 4 libras por animal divididas en 2 tiempos y suero de leche $1 \frac{1}{2}$ litros una vez al día por animal. Hasta alcanzar los 6 meses de edad.

La medición ultrasonografica se efectuó a los 6 meses de edad una semana antes del sacrificio. Se manipularon y se acorralaron los animales para facilitarnos su inmovilización en un área pequeña además se le subministro alimento, con el objetivo de tranquilizarlo para evitar el estrés y posible errores en la medición causados por movimientos excesivos de los animales. Luego los cerdos fueron sometidos a la medición del espesor de grasa subcutánea y el espesor muscular del Longisimo Dorsi utilizando un transductor de $3.5 \mathrm{MHz}$ R60 80 conectado a un aparato de ultrasonido CHISAN-600M, constituyendo estas mediciones la primera etapa de la recogida de datos.. 
El punto anatómico de medición del animal fue rasurado e impregnado de Gel conductor para ultrasonido para facilitar la transmisión de las ondas de ultrasonidos. Las mediciones del espesor de grasa subcutánea y el espesor muscular fueron realizadas en un solo punto de la anatomía de los cerdos vivos. Se midió el espesor del longuísimo dorsi en la última costilla. La medición ultrasonografíca se realizó en la media canal izquierda, posicionando el transductor dorso ventralmente en la superficie previamente rasurada. Las mediciones se repitieron cuatro veces en la misma área, luego se calculó la media de estos valores las cuales fueron utilizadas en la valoración estadística.

Los animales en la edad promedio de 180 días fueron sacrificados en la misma Finca NICALIT-Granja porcina. Se pesaron en forma individual para obtener su peso vivo antes del sacrificio, luego se pesó la canal entera post mortem, posterior a la evisceración se realizó una inspección minuciosa de la canal buscando la presencia de anormalidades específicas. (Abscesos, músculos golpeados), luego tanto la canal izquierda como la derecha fueron despiezadas, obteniéndose el peso de los cortes de carne de calidad específicos, así, cómo el peso de la carne de primera (A), segunda (B), tercera (C) y despojo (D).

Con el conjunto de datos obtenidos se creó la segúnda etapa de la base de datos. Utilizando el programa de Microsoft Excel 2010, se elaboró una hoja de cálculo, donde se plasmaron los valores de todos los parámetros estudiados. Mediciones ultrasonográficas y datos de la canal de los 25 cerdos que se incluyen en esta investigación de campo.

Para el análisis estadístico se utilizó el programa estadístico Micrisoft Excel 2010. En la primera fase del análisis de los datos se estimaron las características estadísticas básicas, media, desviación estándar, mínimo y máximo de las mediciones ultrasonografica del espesor de la grasa dorsal y el espesor del musculo medidos en milímetros, así como la de ambos valores juntos (espesor de grasa dorsal y musculo juntos). Igualmente se estimaron las características estadísticas básicas para los parámetros de la canal obtenidos con el faenado y despiece de los animales: peso vivo, peso de la canal, peso de los distintos cortes cárnicos específicos y el peso de las clases de carne carne de primera $\mathrm{A}$, de segunda $\mathrm{B}$, de tercera $\mathrm{C}$ y despojos $\mathrm{D}$.

Nuestra investigación cuenta con datos primarios en una base de datos, recolectados en campo de forma directa y precisa.

La segunda fase del análisis estadístico fue estimar las correlaciones (r), de Pearson, entre EGD, EM, EGDM o sea las mediciones ultrasonografica y peso vivo, canal, lomo derecho, paleta derecha, aguja, posta de nalga y los tipos de carne Clase A (primera), clase B (segunda), C (tercera) Y D (despojo).

La tercera fase del análisis consistió en determinar el coeficiente de determinación $\left(\mathrm{R}^{2}\right)$ para predecir peso vivo, peso de la canal, lomo derecho, los tipo de carne A (primera), B (segunda), y C (tercera) en relación a las medidas ultrasonográfica.

\section{RESUlTADO Y DISCUSIÓN}

En la tabla 1 se muestran las características estadísticas básicas de las mediciones ultrasonográfica (EGD), (EM), (EGDM). En la cual podemos apreciar el máximo, mínimo, media y desviación, estándar de cada medición realizada. Donde el EGD tiene una media $(12,85 \mathrm{~mm})$, con una desviación estándar $(1,90)$, EM tiene una media $(54,81 \mathrm{~mm})$, desviación estándar $(4.7 \mathrm{~mm})$, y EGDM tiene una media de $(67,66 \mathrm{~mm})$, y una desviación estándar $(6,07)$. 
TABLA 1

Características Estadísticas Básicas de las mediciones ultrasonográfica

\begin{tabular}{lccc}
\hline & $\begin{array}{c}\text { Espesor Grasa Dorsal } \\
(\mathbf{M m})\end{array}$ & $\begin{array}{c}\text { Espesor del musculo } \\
(\mathbf{M m})\end{array}$ & $\begin{array}{c}\text { Espesor Grasa Dorsal y } \\
\text { muscular }(\mathbf{M m})\end{array}$ \\
\hline Máximo & 16.53 & 65.13 & 81.15 \\
Mínimo & 9.53 & 46.3 & 56.53 \\
Media & 12.85 & 54.81 & 67.66 \\
Deviación Estándar & 1.9 & 4.97 & 6.07
\end{tabular}

Fuente: Elaboración propia en base de datos de campo

Podemos observar que la desviación estándar más acusada de los valores de las medidas ultrasonográficas son para el EGDM encontrando la posible explicación en que los cerdos de nuestro experimento son híbridos de las razas Yorkshire, Landrace y Pietrain, razas con diferentes conformación. Los híbridos de la raza Pietrain son más conformados y por ende con mayor masa muscular que de los híbridos de la raza Landrace. La hibridación nos conlleva a una mayor variabilidad genética y debido a eso algunos cerdos pueden variar entre su peso, y algunas medidas zoométrica (anchura, largo del lomo y espesor de grasa), también que en la muestra se utilizaron hembras y machos los cuales acusan un dismorfismo sexual marcado.

La precisión de las mediciones del espesor de grasa dorsal y muscular con ultrasonidos depende de muchos factores, entre los más importantes podemos mencionar: la calidad y potencia del aparato de ultrasonido, la experiencia del operador y el espesor del punto a medir todos estos factores unidos al número de observaciones pueden influenciar los resultados estadísticos.

No obstante los resultados de nuestras mediciones ecográficas sugieren que con esta técnica es posible establecer con poco margen de error las diferencias de conformación y/o desarrollo muscular existentes entre los animales o grupos raciales, pudiendo las mediciones del espesor muscular ser tomadas en cuenta, como factor objetivo a la hora de calificar y/o clasificar las canales porcinas

En la tabla 2 se muestran los parámetros característicos de la canal porcina (cortes de la canal y la clasificación de las carnes según su calidad). En la tabla observamos que el peso vivo máximo encontrado fue de $100 \mathrm{~kg}$, con una canal máxima de $85.45 \mathrm{~kg}$, y a su vez un peso vivo mínimo de $77.27 \mathrm{~kg}$ con una canal mínima de $55 \mathrm{~kg}$, con desviaciones estándares de $6.88 \mathrm{~kg}$ y $7.3 \mathrm{~kg}$ respectivamente, que son bastante bajas. Eso se debe en parte a que los cerdos tuvieron una alimentación estándar y fueron sacrificados prácticamente a la misma edad. Además tuvieron un desarrollo en un mismo ambiente. En general la diferencia viene marcada o determinada como bien lo referíamos anteriormente por la variabilidad genética y las condiciones medioambientales de crianza.

TABLA 2

Características estadísticas básicas de los parámetros de la canal

\begin{tabular}{|c|c|c|c|c|c|c|c|c|c|c|}
\hline & Peso vivo $(\mathrm{kg})$ & $\begin{array}{c}\text { Canal entera } \\
(\mathrm{kg})\end{array}$ & $\begin{array}{c}\text { Lomo } \\
\text { derecho }(\mathrm{kg})\end{array}$ & $\begin{array}{c}\text { Paleta } \\
\text { derecha }(\mathrm{kg})\end{array}$ & Aguja (kg) & $\begin{array}{c}\text { Posta nalga } \\
(\mathrm{kg})\end{array}$ & Clase A (kg) & $\begin{array}{l}\text { Clase } \\
\text { B (kg) }\end{array}$ & Clase C (kg) & Clase D (kg) \\
\hline Máximo & 100 & 85.45 & 9.54 & 8.18 & 4.09 & 7.72 & 27.3 & 25.4 & 23.63 & 3.16 \\
\hline Mínimo & 77.27 & 55 & 6.81 & 3.63 & 1.59 & 4.09 & 18.6 & 13.6 & 15.45 & 1.05 \\
\hline Media & 90.42 & 73.32 & 8.87 & 5.98 & 2.18 & 5.04 & 22.47 & 19.21 & 18.9 & 1.76 \\
\hline Deviación Esté & 6.88 & 7.73 & 0.69 & 1.25 & 0.6 & 0.88 & 2.1 & 2.99 & 1.68 & 0.59 \\
\hline
\end{tabular}

Fuente: Elaboración propia en base de datos de campo.

Entre la media del peso vivo $(90.42 \mathrm{~kg})$ y la media de la canal entera $(73.32 \mathrm{~kg})$ existe una diferencia de valores, que se debe a los procedimientos realizados al sacrificio como: pérdida de sangre, y la evisceración.

El más alto coeficiente de correlación lineal encontrado fue entre el peso vivo y la canal entera $(r=0.85)$, lo que era de esperarse, debido a que la diferencia de peso entre ambas variables se reduce al peso de las vísceras y la sangre, igualmente entre el peso vivo y el lomo derecho $(r=0.68)$ ya que el lomo representa una pieza de 
gran peso, por lo que guarda una relación directa con el peso vivo. Altos coeficientes de correlación fueron encontrados entre el espesor de la grasa dorsal y la canal entera $(\mathrm{r}=0.67)$, así como también entre el espesor de la grasa dorsal y el peso vivo $(\mathrm{r}=0.53)$ representando estos los resultados más importantes en nuestro estudio estos resultados son similares a los presentados por Demo, P. et al. (1993), Mejía G et al (1999) Y Boland M et al (1995). Altos coeficientes de correlación fueron encontrados entre el lomo derecho y la carne clase A $(\mathrm{r}=0.80)$, entre la paleta derecha y clase $\mathrm{B}(\mathrm{r}=0.88)$, entre posta de nalga con la clase A y clase $\mathrm{B}(\mathrm{r}=0.75 \mathrm{y}$ 0.70 respectivamente). El resto de correlaciones fueron bajas o no significativas TABLA 3.A.

TABLA 3A

Correlaciones de Grasa Dorsal ( $\mathrm{mm}$ ) Fuente: Elaboración propia en base de datos de campo.

\begin{tabular}{|c|c|c|c|c|c|c|c|c|c|c|c|}
\hline & $\begin{array}{c}\text { Espesor } \\
\text { Grasa Dorsal }\end{array}$ & $\begin{array}{l}\text { Peso } \\
\text { vivo }\end{array}$ & $\begin{array}{l}\text { Canal } \\
\text { entera }\end{array}$ & $\begin{array}{c}\text { Lomo } \\
\text { derecho }\end{array}$ & $\begin{array}{c}\text { Paleta } \\
\text { derecha }\end{array}$ & Aguja & $\begin{array}{l}\text { Posta } \\
\text { nalga }\end{array}$ & $\begin{array}{c}\text { Clase } \\
\text { A }\end{array}$ & $\begin{array}{c}\text { Clase } \\
B\end{array}$ & $\begin{array}{c}\text { Clase } \\
\text { C }\end{array}$ & $\begin{array}{c}\text { Clase } \\
D\end{array}$ \\
\hline Espesor Grasa & 1 & & & & & & & & & & \\
\hline Peso vivo & 0.538 & 1 & & & & & & & & & \\
\hline Canal entera & 0.674 & 0.853 & 1 & & & & & & & & \\
\hline Lomo derecho & 0.293 & 0.688 & 0.467 & 1 & & & & & & & \\
\hline Paleta derecha & 0.038 & 0.047 & -0.15 & 0.415 & 1 & & & & & & \\
\hline Aguja & -0.259 & 0.025 & -0.187 & -0.018 & -0.232 & 1 & & & & & \\
\hline Posta nalga & -0.194 & 0.121 & -0.225 & 0.391 & 0.331 & 0.638 & 1 & & & & \\
\hline Clase A & 0.022 & 0.458 & 0.195 & 0.808 & 0.422 & 0.33 & 0.755 & 1 & & & \\
\hline Clase B & -0.082 & 0.076 & -0.228 & 0.463 & 0.888 & 0.201 & 0.709 & 0.659 & 1 & & \\
\hline Clase C & 0.098 & 0.451 & 0.193 & 0.572 & 0.204 & 0.421 & 0.717 & 0.765 & 0.487 & 1 & \\
\hline Clase D & -0.299 & -0.19 & -0.461 & 0.147 & 0.35 & 0.169 & 0.576 & 0.318 & 0.5 & 0.379 & 1 \\
\hline
\end{tabular}

Fuente: Elaboración propia en base de datos de campo.

TABLA 3B

Correlación Espesor Muscular ( $\mathrm{mm}$

\begin{tabular}{|c|c|c|c|c|c|c|c|c|c|c|c|}
\hline & $\begin{array}{l}\text { Espesor } \\
\text { Muscular }\end{array}$ & Peso vivo & $\begin{array}{c}\text { Canal } \\
\text { entera }\end{array}$ & $\begin{array}{c}\text { Lomo } \\
\text { derech }\end{array}$ & $\begin{array}{r}\text { Paleta } \\
\text { derec }\end{array}$ & Aguja & $\begin{array}{l}\text { Posta } \\
\text { nalga }\end{array}$ & $\begin{array}{c}\text { Clase } \\
\text { A }\end{array}$ & $\begin{array}{c}\text { Clase } \\
B\end{array}$ & $\begin{array}{c}\text { Clase } \\
C\end{array}$ & $\begin{array}{c}\text { Clase } \\
D\end{array}$ \\
\hline \multicolumn{12}{|l|}{ Espesor } \\
\hline $\begin{array}{l}\text { Muscular } \\
(\mathrm{mm})\end{array}$ & 1 & & & & & & & & & & \\
\hline Peso vivo & 0.293 & 1 & & & & & & & & & \\
\hline Canal entera & 0.286 & 0.853 & 1 & & & & & & & & \\
\hline $\begin{array}{l}\text { Lomo } \\
\text { derecho }\end{array}$ & 0.223 & 0.688 & 0.467 & 1 & & & & & & & \\
\hline $\begin{array}{l}\text { Paleta } \\
\text { derecha }\end{array}$ & -0.148 & 0.047 & -0.15 & 0.415 & 1 & & & & & & \\
\hline Aguja & 0.219 & 0.025 & -0.187 & -0.018 & -0.232 & 1 & & & & & \\
\hline Posta nalga & 0.103 & 0.121 & -0.225 & 0.391 & 0.331 & 0.638 & 1 & & & & \\
\hline Clase A & 0.225 & 0.458 & 0.195 & 0.808 & 0.422 & 0.33 & 0.755 & 1 & & & \\
\hline Clase B & -0.07 & 0.076 & -0.228 & 0.463 & 0.888 & 0.201 & 0.709 & 0.659 & 1 & & \\
\hline Clase C & 0.031 & 0.451 & 0.193 & 0.572 & 0.204 & 0.421 & 0.717 & 0.765 & 0.487 & 1 & \\
\hline Clase D & -0.294 & -0.19 & -0.461 & 0.147 & 0.35 & 0.169 & 0.576 & 0.318 & 0.5 & 0.379 & 1 \\
\hline
\end{tabular}

Fuente: Elaboración propia en base de datos de campo.

Al correlacionar el espesor muscular con los parámetros de la canal aquí estudiados encontramos correlaciones de medias a bajas $(\mathrm{r}=0.29$ - 0.22) TABLA. 3. B. Al correlacionar el espesor de la grasa subcutánea y espesor muscular juntos con los parámetros de la canal encontramos correlaciones de medias a altas $(r=0.44-0.40$ correspondientes a canal entera y peso vivo respectivamente) estos valores son comparables con los encontrados por (Jogal S.M, Kennedy B.W, 1987), (McLaren D.; McKeithf F.; Novakofskij J1989)TABLA 3. C. 
TABLA 3C

Correlación Grosor grasa subcutánea y espesor muscular

(mm) Fuente: Elaboración propia en base de datos de campo.

\begin{tabular}{|c|c|c|c|c|c|c|c|c|c|c|}
\hline & $\begin{array}{c}\text { Grosor de la } \\
\text { grasa } \\
\text { subcutánea y } \\
\text { espesor } \\
\text { muscular }\end{array}$ & Peso vivo & $\begin{array}{l}\text { Canal } \\
\text { entera }\end{array}$ & $\begin{array}{c}\text { Lomo } \\
\text { derecho }\end{array}$ & $\begin{array}{c}\text { Paleta } \\
\text { derecha }\end{array}$ & Aguja & $\begin{array}{l}\text { Posta } \\
\text { nalga }\end{array}$ & Clase $A$ & $\begin{array}{c}\text { Clase } \\
B\end{array}$ & Clase C Clase D \\
\hline $\begin{array}{l}\text { Grosor de la } \\
\text { grasa } \\
\text { subcutánea y } \\
\text { espesor } \\
\text { muscular } \\
\text { (mm) }\end{array}$ & 1 & & & & & & & & & \\
\hline Peso vivo & 0.409 & 1 & & & & & & & & \\
\hline Canal entera & 0.446 & 0.853 & 1 & & & & & & & \\
\hline Lomo derecho & 0.275 & 0.688 & 0.467 & 1 & & & & & & \\
\hline Paleta derech: & -0.109 & 0.047 & -0.15 & 0.415 & 1 & & & & & \\
\hline Aguja & 0.098 & 0.025 & -0.187 & -0.018 & -0.232 & 1 & & & & \\
\hline Posta nalga & 0.023 & 0.121 & -0.225 & 0.391 & 0.331 & 0.638 & 1 & & & \\
\hline Clase A & 0.191 & 0.458 & 0.195 & 0.808 & 0.422 & 0.33 & 0.755 & 1 & & \\
\hline Clase B & -0.083 & 0.076 & -0.228 & 0.463 & 0.888 & 0.201 & 0.709 & 0.659 & 1 & \\
\hline Clase $\mathrm{C}$ & 0.056 & 0.451 & 0.193 & 0.572 & 0.204 & 0.421 & 0.717 & 0.765 & 0.487 & 1 \\
\hline Clase D & -0.335 & -0.19 & -0.461 & 0.147 & 0.35 & 0.169 & 0.576 & 0.318 & 0.5 & 0.379 \\
\hline
\end{tabular}

Fuente: Elaboración propia en base de datos de campo.

Se evaluó la posibilidad de predecir algunos parámetros de la canal aquí estudiados (carne clase A, B Y C) a partir de las mediciones ultrasonográfica, integrada en modelos de ecuaciones de regresión lineal con resultados variados. El coeficiente de determinación del modelo de predicción de regresión lineal más elevado fue el de la medición ultrasonografía del espesor de grasa dorsal $\left(\mathrm{R}^{2}=0.55\right.$ con una correlación múltiple de 0.74). Estos resultados son comparables con los encontrados por (McLaren D.; McKeithf F.; Novakofskij J1989), (Youssao I.; Verleyen V.; Verleyen P, 2002) pero inferiores a los obtenidos por Demo et al 1993) TABLA 4 (Ver Anexo).

En la tabla 5 (ver Anexo) se muestran los resultados obtenidos en el modelo de regresión lineal utilizando como variable independiente el espesor muscular, la correlación múltiple obtenida fue de $(r=0.48)$ y el coeficiente de determinación fue de $\left(\mathrm{R}^{2}=0.23\right)$. En el caso de grosor grasa subcutánea y espesor muscular juntos los resultados fueron similares $(r=0.50$ y $R 2=0.25$ respectivamente) comparables con los resultados de C.J. López-Bote 2006) $\left(\mathrm{R}^{2}=0,23-0,40\right)$ y (A.P Sather; H.T Fredeen 1982) $\left(\mathrm{R}^{2}=0,35,0,35\right)$.

Existe diferencia en los resultados de los modelos si se integra o no a estos el peso vivo. En este sentido Walder et at, (1992) indican que la inclusión del peso vivo antes de sacrificio en los modelos de predicción produce un aumento mínimo de R2 del 2\% mientras que SLONIEWSKI et al (1997) dicen haber encontrado un aumento mínimo del $\mathrm{R}^{2}$ del $5 \%$ en comparación con los modelos donde se incluían solo las mediciones ecográficas. En nuestros modelos se incluyó el peso vivo.

Los cálculos de predicción en nuestro trabajo indican que el modelo de regresión lineal donde se utilizó el espesor de grasa subcutánea tiene una capacidad de predicción moderada. La capacidad de predicción para los restantes modelos fue baja.

\section{Conclusiones}

El espesor de grasa subcutánea del longuísimo dorsi medido en la última costilla de la media canal izquierda del cerdo, puede ser considerado como buen predictor del peso de la canal entera, y el corte de primera lomo de la canal porcina. 
No obstante sería recomendable continuar en la investigación, repetir estudios similares con mayor número de animales para optimizar las ecuaciones de predicción de la composición de la canal porcina.

En la Bioeconomía global la Eco intensificación de la producción porcina utilizando método de ultrasonografía para mejorar el rendimiento destaca la importancia de la Bioeconomía porcina en este sector productivo (Zúniga-González, C. A. et.al (2014)

\section{LITERATURA CITADA}

Blanco Roa N., huba J., Hetenyi L., Oravcová A. (2008): Estimación in vivo de la composición de la canal en bovinos utilizando mediciones ultrasonografica; universitas 1. 2008 pag 58-63. https://doi.org/10.5377/ universitas.v2i1.1645

López-Bote., A. Daza.: Effect of feeding system on the growth and carcass characteristics of Iberian pigs, and the use of ultrasound to estimate yields of joints. Meat science. 72, 2006, p 1-8. https://doi.org/10.1016/ j.meatsci.2005.04.031

Sather, A. P., H. T. Fredeen, A. H. Martin.: Live animal evaluation of two ultrasonic probes as estimators of subcutaneous backfat and carcass composition in pigs. 1982; Canadian Journal of Animal Science P.82-114.

López G., Rubio M. (1998). Tecnología para la evaluación objetiva de las canales de animales de abasto. Vet.Méx. 29, 1998 , p. 287.

Londoño J., Velásquez C. (2013): Clasificación y valoración de la calidad de canales porcinas en Colombia, monografía para optar al título de especialista en gerencia agropecuaria, 2013, p. 70.

Gérard D.(2001) Clasificación de las canales porcinas en Francia y en Europa. Memorias $9^{\circ}$ Seminario Nacional de Desenvolvimiento de Suinocultura. InstitutTechnique du Porc Francia, 2001 25-27 de abril de 2001. P 6-9.

Jogal S.M, Kennedy B.W: (1987). Evaluación de los equipos de medición eléctricos en la predicción de la composición de la canal en cerdos vivo, animal production, 1987, 45, P 97-102

Mejía G.C.A.; Montaño B.M.; Velázquez M.P.A. y Cuarón, I.J.A.: Estimación in vivo del rendimiento de las canales porcinas mediante ultrasonografía. Téc. Pecu. Méx. 1999, 37:2. P 31.

McLaren D.; McKeith F.; Novakofski J. Prediction of CarcassCharacteristics at Market Weightfrom Serial Real-Time Ultrasound Measures of Backfat and Loin Eye Area in the Growing Pig. Journal of Animal Science.67, 1989, P, 1657-1667.

Swantek P.; Crenshaw J.; Marchello M.; Lukaski H.(1992). Impedancia bioeléctrica: un método no destructivo para determinar la masa libre de grasa de cerdo de mercado y cerdo cadáveres vivos. J Animsci. 70.1992. P 77-169.

Boland M.; Foster K.; Schinckel.; Wagner J.; Chen W.; Berg E.; Forrest J.(1995) Técnicas de evaluación de la canal alternativa: Las diferencias en las predicciones de valor. J anim sci.73, 1995. P 44.

Ordenes L.:(2005). Evaluación del espesor de la grasa dorsal y peso vivo en jabalí puro y en mestizos desde las 17 hasta las 39 semanas de edad, memoria presentada a la facultad de medicina veterinaria de la universidad de concepción para optar al título de médico veterinario; Chillan-Chile, 2005, P 6.

Higbie A.; Bidner T.; Matthews J.; Southern L.; Page T.; Persica M.: Sanders M.; Monlezun C. (2002). Prediction of swinecarcass composition by total body electrical conductivity. J. Anim. Sci. 2002. 80 P.113.

Youssao I.; Verleyen V.; Verleyen P.: Prediction of carcass lean content by real-time ultrasound in Pietrain and negative stress Pietrain. Animal Science. 2002, 75 P 25. https://doi.org/10.1017/S1357729800052796

Definición canal porcina [en línea] http://www.uco.es/zootecniaygestion/ [citado el 20 de marzo de 2015]

Escáneres de ultrasonido [citado en línea] http://www.cancerquest.org/index.cfm?page=3422\&lang [citado el 19 de marzo de 2015]

Zúniga-González, C. A., Durán Zarabozo, O., Dios Palomares, R., Sol Sánchez, A., Guzman Moreno, M. A., Quiros, O., \& Montoya Gaviria, G. D. J. (2014). Estado del arte de la bioeconomía y el cambio climático ISBN 978-99924-28-39-9, ISBN 978-99924-28-40-5, pp. 20-329) Editorial Universitaria UNAN León. 
Revista Iberoamericana de Bioeconomía y Cambio Climático, 2019, 5(10), Agosto-Diciembre, ISSN: 241...

Universo porcino (2015). Consumo mundial de carne porcina [en línea] http://www.aacporcinos.com.ar [citado el 23 de marzo de 2015]

Central American Data (2015). El principal productor y consumidor mundial de carne [en línea] http:// www.centralamericadata.com [citado el 20 de marzo de 2015]

UCO (2015). Definición canal porcina [en línea] http://www.uco.es/zootecniaygestion/ [citado el 20 de marzo de 2015]

ESCI (2015).Escáneres de ultrasonido [citado en línea] http://www.cancerquest.org/index.cfm?page=3422\&lang [citado el 19 de marzo de 2015]

\section{REFERENCIAS}

Walder D N, Dikeman M E, Schalles R R, Olson W G, Houghton P L, Unruh J A and Corah L R 1992 Validation of Real - time Ultrasound technology for Predicting Fat thicknesses, Longissimus Muscle Areas and Composition of brangus bulls from 4 Months to 2 Years of Age. Journal of Animal Science 70: 3044 - 3054, disponible en: http://www.journalofanimalscience.org/content/70/10/3044.full.pdf

Dios Palomares, R. , Alcaide, D., Diz, J., Jurado, M., Prieto, A., Morantes, M., \& Zúñiga, C. A. (2015). Análisis de la eficiencia de sistemas agropecuarios en América latina y el Caribe mediante la incorporación de aspectos ambientales. Revista Cientifica de la Facultad de Ciencias Veterinarias de la Universidad del Zulia, 25(1), 43-50.

Sloniewski, P., \& Zielinski, P. (1997). Remote effect of brain retraction on regional cerebral blood flow and cerebrovascular reserve on single photon emission computed tomography. Surgical Neurology, 48(5), 511-513.

Demo P, Krška P, Poltársky J and Borecký A 1993 The use of an echocamera for in vivo prediction of some carcass characteristics in pig. Živoc 38: 645 - 654. 
Santiago Hernández Zapata, et al. Estimación in vivo de la canal porcina por el método de ultrason...

\section{Anexos}

\begin{tabular}{|c|c|c|c|c|c|c|c|c|c|}
\hline \multicolumn{2}{|l|}{ Estadisticas de la regresión } & & & & & & & & \\
\hline Coeficiente de correlación multiple & 0.744 & & & & & & & & \\
\hline Coeficiente de determinación $R^{\wedge} 2$ & 0.553 & & & & & & & & \\
\hline$R^{\prime} / 2$ ajustado & 0.404 & & & & & & & & \\
\hline Error típico & 1.442 & & & & & & & & \\
\hline Observaciones & 25 & & & & & & & & \\
\hline \multicolumn{10}{|l|}{ ANÁLISIS DE VA RIA NZA } \\
\hline & $\begin{array}{c}\text { Grados de } \\
\text { libertad }\end{array}$ & $\begin{array}{c}\text { Suma de } \\
\text { cuadrados }\end{array}$ & $\begin{array}{c}\text { Promedio } \\
\text { de los } \\
\text { cuadrados }\end{array}$ & $F$ & \multicolumn{2}{|c|}{ Valor critico de $F$} & & & \\
\hline Regresión & 6 & 46.287 & 7.714 & 3.713 & & 0.014 & & & \\
\hline Residuos & 18 & 37.404 & 2.078 & & & & & & \\
\hline \multirow[t]{2}{*}{ Total } & 24 & 83.69 & & & & & & & \\
\hline & $\begin{array}{c}\text { Coeficient } \\
\text { es }\end{array}$ & $\begin{array}{l}\text { Error } \\
\text { tipico }\end{array}$ & $\begin{array}{c}\text { Estadistic } \\
o t\end{array}$ & \multicolumn{2}{|c|}{ Probabilidad } & Inferior $95 \%$ & $\begin{array}{c}\text { Superior } \\
95 \%\end{array}$ & $\begin{array}{l}\text { Inferior } \\
95.0 \%\end{array}$ & $\begin{array}{l}\text { Superio } \\
\text { r.95.0\% }\end{array}$ \\
\hline Intercepción & 0.649 & 4.41 & 0.147 & & 0.885 & -8.616 & 9.913 & -8.616 & 9.913 \\
\hline Pesovivo & -0.093 & 0.114 & -0.82 & & 0.423 & -0.333 & 0.146 & -0.333 & 0.146 \\
\hline Canal entera & 0.238 & 0.09 & 2.645 & & 0.016 & 0.049 & 0.427 & 0.049 & 0.427 \\
\hline Lomo derecho & 0.891 & 0.951 & 0.937 & & 0.361 & -1.107 & 2.889 & -1.107 & 2.889 \\
\hline Clase A & -0.611 & 0.355 & -1.721 & & 0.102 & -1.358 & 0.135 & -1.358 & 0.135 \\
\hline Clase B & 0.224 & 0.153 & 1.465 & & 0.16 & -0.097 & 0.546 & -0.097 & 0.546 \\
\hline Clase C & 0.252 & 0.294 & 0.855 & & 0.404 & -0.367 & 0.87 & -0.367 & 0.87 \\
\hline
\end{tabular}

TABLA 4

Regresión Espesor Grasa Dorsal

fuente: Elaboración propia

\begin{tabular}{|c|c|c|c|c|c|c|c|c|}
\hline \multicolumn{2}{|l|}{ Estadisticas de la regresión } & & & & & & & \\
\hline Coeficiente de correlación múltiple & 0.4846 & & & & & & & \\
\hline Coeficiente de determinación $\mathbf{R}^{\wedge} \mathbf{2}$ & 0.2349 & & & & & & & \\
\hline $\mathrm{R}^{\wedge} \mathbf{2}$ ajustado & -0.0202 & & & & & & & \\
\hline Error tipico & 4.9207 & & & & & & & \\
\hline Olservaciones & 25 & & & & & & & \\
\hline \multicolumn{9}{|l|}{ ANÁLISIS DEVARIANZA } \\
\hline & \begin{tabular}{|c|} 
Grados \\
de \\
libertad \\
\end{tabular} & $\begin{array}{c}\text { Suma de } \\
\text { cuadrados }\end{array}$ & \begin{tabular}{|c|}
$\begin{array}{c}\text { Promedio } \\
\text { de los } \\
\text { cuadrados }\end{array}$ \\
\end{tabular} & $F$ & Valor crítico de $F$ & & & \\
\hline Regresión & 6 & 133.792 & 22.299 & 0.921 & 0.503 & & & \\
\hline Residuos & 18 & 435.839 & 24.213 & & & & & \\
\hline \multirow[t]{2}{*}{ Total } & 24 & 569.632 & & & & & & \\
\hline & \begin{tabular}{|c|}
$\begin{array}{c}\text { Coefici } \\
\text { entes }\end{array}$ \\
\end{tabular} & $\begin{array}{l}\text { Error } \\
\text { típico }\end{array}$ & \begin{tabular}{|c|} 
Estadistico \\
$t$ \\
\end{tabular} & \begin{tabular}{|l|} 
Probab \\
ilidad \\
\end{tabular} & Inferior $95 \%$ & \begin{tabular}{c|} 
Superior \\
$95 \%$ \\
\end{tabular} & $\begin{array}{c}\text { Inferior } \\
95.0 \%\end{array}$ & $\begin{array}{c}\text { Superior } \\
95.0 \% \\
\end{array}$ \\
\hline Intercepción & 42.01 & 15.053 & 2.791 & 0.012 & 10.386 & 73.634 & 10.386 & 73.634 \\
\hline Peso vivo & 0.266 & 0.389 & 0.685 & 0.502 & -0.551 & 1.083 & -0.551 & 1.083 \\
\hline Canal entera & -0.025 & 0.307 & -0.081 & 0.937 & -0.669 & 0.62 & -0.669 & 0.62 \\
\hline Lomo derecho & -2.225 & 3.246 & -0.686 & 0.502 & -9.045 & 4.594 & -9.045 & 4.594 \\
\hline Clase A & 2.054 & 1.213 & 1.693 & 0.108 & -0.494 & 4.602 & -0.494 & 4.602 \\
\hline Clase B & -0.512 & 0.523 & -0.979 & 0.341 & -1.611 & 0.587 & -1.611 & 0.587 \\
\hline Clase C & -1.381 & 1.004 & -1.375 & \begin{tabular}{|l|}
0.186 \\
\end{tabular} & -3.491 & 0.729 & -3.491 & 0.729 \\
\hline
\end{tabular}

TABLA 5

Regresión Espesor Musculo 